\title{
NOVA TECNOLOGIA DE BRANQUEAMENTO DE CELULOSE ADAPTADA AO FECHAMENTO DO CIRCUITO DE ÁGUA ${ }^{1}$
}

\author{
Marcelo Moreira Costa ${ }^{2}$, Jorge Luiz Colodette ${ }^{3}$, Alexandre Landim ${ }^{4}$, Cláudio Mudado Silva ${ }^{3}$ e Ana \\ Márcia Macêdo Ladeira Carvalho ${ }^{3}$
}

\begin{abstract}
RESUMO - A Celulose Nipo-Brasileira é uma das maiores produtoras de celulose kraft branqueada de eucalipto no Brasil. Produz 860.000 tsa/ano em duas linhas, que são equipadas com digestores contínuos. Ambas as linhas fabricam polpa ECF (Elemental Chlorine Free) com as seqüências $\mathrm{D}_{\text {hot }}(\mathrm{EOP}) \mathrm{D}(\mathrm{EP}) \mathrm{D}$ e D(EOP)DP, respectivamente, na linha 1 e 2. A fábrica tem tratamento do efluente com lodo ativado com dois tanques de aeração com capacidade para $20.000 \mathrm{~m}^{3}$, equipados com aeradores superficiais seguidos por quatro clarificadores secundários (dois para cada reator). Nas últimas décadas, a fábrica tem otimizado e vem mudando seus processos, a fim de melhorar a preservação ambiental. Com o objetivo de reduzir o volume de efluente, DQO e carga de AOX, a seqüência $\mathrm{A}_{\text {hot }}(\mathrm{EOP}) \mathrm{D}(\mathrm{PO})$ proposta foi avaliada em testes laboratoriais, com reciclagem de filtrado parcial. Este artigo propôs a reciclagem de filtrado, que reduz o volume de efluente da fábrica em $9 \mathrm{~m}^{3} / \mathrm{tsa}$ (tonelada secada ao ar), isto é, mais ou menos 50\% do total. O filtrado recuperado é parcialmente desviado para o ciclo de recuperação e para o estágio de deslignificação oxigênio. A reutilização do filtrado $A_{\text {hot }}$ no ciclo de recuperação é para substituir os filtrados, atualmente usados para lavar lama de cal e “dregs”. O impacto dos NPEs no ciclo de cálcio não foi significante. Essa estratégia permitiu uma recuperação de carga alcalina de $12 \mathrm{~kg} \mathrm{NaOH} /$ tsa de polpa, que, do contrário, seria perdida. A branqueabilidade da polpa e a sua qualidade não foram afetadas significativamente. O efluente descartado, proveniente das etapas $\mathrm{D}(\mathrm{PO})$, mostrou-se com baixas cargas de cor, de DQO, de AOX e de uma boa biodegradabilidade (DBO5/DQO).
\end{abstract}

Palavras-chave: Polpa kraft branqueada de Eucalyptus, fechamento parcial de circuito, estabilidade da polpa, consumo de dióxido de cloro, qualidade da polpa e custo operacional.

\section{A NOVEL BLEACHING TECHNOLOGY ADAPTED TO PARTIAL BLEACH PLANT CLOSURE}

\begin{abstract}
Celulose Nipo-Brasileira is one of the largest eucalyptus bleached kraft pulp mills in Brazil. It produces 860,000 tpy in two lines that are equipped with continuous digesters. Line 1 and line 2 produces ECF (Elemental Chlorine Free) pulp with the sequences: Dhot(EOP)D(EP)D and D(EOP)DP, respectively. The mill has an activated sludge effluent treatment plant comprised of two aeration tanks with 20,000 m3 and it is equipped with superficial aerators followed by four secondary clarifiers (two for each reactor). In the last decades, CENIBRA has been optimizing and changing its processes in order to improve environmental performance. Aiming at decreasing effluent volume, COD and AOX loads, the Ahot(EOP)D(PO) sequence
\end{abstract}

\footnotetext{
${ }^{1}$ Recebido em 27.07.2004 e aceito para publicação em 10.11.2005.

${ }^{2}$ Veracel Celulose S.A.

${ }^{3}$ Departamento de Engenharia Florestal da Universidade Federal de Viçosa, Viçosa-MG. E-mail: <colodett@ufv.br>.

${ }^{4}$ Cenibra S/A.
} 
has been proposed and evaluated in lab-scale trials, with partial filtrate recycling. This paper proposes a filtrate recycling scheme that reduces bleach plant effluent volume by 9 m3/adt (air dry ton), i.e., about $50 \%$ of the total. The filtrate saved is partially diverted to the recausticizing cycle and to post oxygen washers. The reutilization of the Ahot filtrate in the recausticizing cycle to replace the filtrates currently used to wash lime mud and dregs was shown suitable. The impact of NPE's in the calcium cycle was not significant. This strategy allowed a recovery of c.a. $12 \mathrm{~kg} \mathrm{NaOH} /$ adt of pulp that would otherwise be wasted. Pulp bleachability and quality was not significantly affected. The discharged effluent coming from $D(P O)$ stages (about $50 \%$ ) showed low color, COD and AOX loads, and good bio-treatability (DBO5/COD).

Keywords: Bleached kraft Eucalyptus pulp, partial bleach plant closure, pulp stability, chlorine dioxide consumption, pulp quality and operational costs.

\section{INTRODUÇÃO}

Nas últimas décadas, a indústria de celulose vem modificando e otimizando os seus processos fabris, com o objetivo de melhorar a sua capacidade de produção, a qualidade de seus produtos e, sobretudo, o seu desempenho ambiental. Mundialmente, existe uma consciência crescente da escassez de água, o que inaugura um novo período de abordagens voltadas para as questões ambientais que dominam os cenários político, jurídico, econômico e administrativo de vários processos industriais.

Dentre os diversos estágios que compõem o processo Kraft, a planta de branqueamento constitui a maior fonte de geração de efluentes líquidos. Geralmente, esses efluentes contêm altos teores de matéria orgânica, alta cor e compostos organoclorados, bem como residuais de produtos químicos usados na planta de branqueamento. Portanto, alternativas que buscam a reutilização dos filtrados do branqueamento, parcial ou totalmente, promovem impacto significativo de redução do consumo de água, da geração de efluentes e, sobretudo, da poluição ambiental. No entanto, a reutilização desses filtrados do branqueamento é desafiante, por causa do acúmulo de Non-Process Elements (NPEs) no sistema. Maior atenção tem sido dada aos seguintes elementos: $\mathrm{Cl}, \mathrm{K}, \mathrm{P}, \mathrm{Si}, \mathrm{Al}, \mathrm{Mg}$, Ca, Ba, Mn, Fe e Cu (VÄLTTILÄ et al., 1996; ULGREN, 1997; GLEADOW et al., 1997).

Os principais efeitos dos NPEs que dificultam o fechamento de circuito são: aumento da taxa de decomposição de agentes de branqueamento derivado do oxigênio, formação de incrustações, aumento da taxa de corrosão de equipamentos e tubulações e acúmulo no sistema de recuperação, incremento da carga de dregs, grits e outros (TRAN et al., 1990; LINDBERG et al., 1994), além de causar prejuízos à qualidade da polpa. Costa et al. (2000) relataram que, apesar da existência dos NPEs nos filtrados de branqueamento, existem elementos desejáveis como o Na. Esse fato deve ser levado em consideração numa avaliação econômica das seqüências de branqueamento, já que o processo necessita de reposições periódicas desse elemento no sistema de recuperação.

Com a finalidade de reduzir o consumo de hidróxido de sódio no processo, a carga orgânica (DQO) e o volume dos efluentes enviados à estação de tratamento secundário, foi proposta a utilização da seqüência $\mathrm{A}_{\text {hot }}(\mathrm{EOP}) \mathrm{D}(\mathrm{PO})$, com fechamento parcial de circuito de águas. Essa seqüência foi a alternativa considerada de melhor potencial realizada em estudo prévio (COSTA et al., 2001). Nessa seqüência, praticamente a metade do volume dos filtrados usados na lavagem da polpa é potencialmente reutilizável no processo como água industrial.

O principal objetivo deste trabalho foi avaliar o impacto do fechamento parcial dos circuitos de água da seqüência $A_{\text {hot }}$ (EOP)D(PO) na branqueabilidade da polpa, medida pelo consumo de reagentes químicos e na qualidade do efluente gerado. Avaliou-se, também, o potencial de reutilização dos filtrados do estágio $\mathrm{A}_{\text {hot }}$ e (EOP) no processo.

\section{EXPERIMENTAL}

\subsection{Material}

Foram utilizadas três amostras de polpa Kraft- $\mathrm{O}_{2}$ de Eucalyptus spp (Quadro 1) originadas de cozimentos modificados (ITC e Kobudo ${ }^{\text {mari }}$ - Tecnologia Kvaerner). 
Quadro 1 - Características das amostras de polpa kraft- $\mathrm{O}_{2}$ industriais Table I-Characteristics of the three oxygen delignified. kraft pulp samples

\begin{tabular}{|c|c|c|c|}
\hline \multirow[t]{2}{*}{ Parâmetros Analisados } & \multicolumn{3}{|c|}{ Identificação das Amostras } \\
\hline & F - ITC & $\mathrm{J}-\mathrm{Kobudo}^{\text {mari }}$ & $\mathrm{H}-\mathrm{Kobudo}^{\text {mari }}$ \\
\hline Número kappa & 8,3 & 7,1 & 8,3 \\
\hline HexA's, mmol/kg & 38,8 & 36,8 & 34,0 \\
\hline Viscosidade, mPa.s & 19,2 & 19,3 & 19,6 \\
\hline Alvura, \% ISO & 49,0 & 56,5 & 49,1 \\
\hline DQO da polpa, $\mathrm{kg} \mathrm{O}_{2} / \mathrm{t}$ & 26,0 & 4,19 & 10,2 \\
\hline Perda alcalina, kg NaOH/t & 11,0 & 5,8 & 11,3 \\
\hline Teor cinza, \% & 1,53 & 0,58 & 0,53 \\
\hline \multicolumn{4}{|l|}{ Conteúdo de metais, g/t } \\
\hline $\mathrm{Ca}$ & 1.258 & 1.290 & 666 \\
\hline $\mathrm{Mg}$ & 38,7 & 139 & 34,0 \\
\hline $\mathrm{Mn}$ & 8,80 & 3,70 & 1,04 \\
\hline $\mathrm{Fe}$ & 24,4 & 4,41 & 6,92 \\
\hline $\mathrm{Cu}$ & 0,11 & 0,21 & 1,17 \\
\hline
\end{tabular}

\subsection{Métodos}

\subsubsection{Estratégias de recirculação dos filtrados da seqüência $A_{\text {hot }}(E O P) D(P O)$}

Neste estudo foi utilizada a polpa kraft- $\mathrm{O}_{2}$ identificada pela letra $H$ (Quadro 1). A estratégia de recirculação de filtrados para a seqüência $\mathrm{A}_{\text {hot }}(\mathrm{EOP}) \mathrm{D}(\mathrm{PO})$ é mostrada na Figura 1. A simulação baseou-se numa seqüência que tem elementos de lavagem após os estágios $A_{\text {hot }}$, (EOP) e D, bem como prensas após a pré- $\mathrm{O}_{2}$ e o estágio (PO). $\mathrm{O}$ fator de diluição nos filtros e na prensa dessa seqüência de branqueamento foi fixado em 1,67 m³ t. Após a prensa da pré- $\mathrm{O}_{2}$, foi necessário um volume de $6,67 \mathrm{~m}^{3} / \mathrm{t}$ de filtrado para ajuste de consistência de 30 para $10 \%$. Nesse ponto, o ajuste de consistência foi realizado com o próprio filtrado do estágio $A_{\text {hot }}$. No entanto, existe ainda um volume disponível de 4,0 $\mathrm{m}^{3} / \mathrm{t}$. Desse volume, 3,89 $\mathrm{m}^{3} / \mathrm{t}$ foram projetados para serem utilizados na caustificação na lavagem da lama de cal $\left(\sim 2,78 \mathrm{~m}^{3} / \mathrm{t}\right)$ e do dregs $\left(\sim 1,11 \mathrm{~m}^{3} / \mathrm{t}\right)$. O restante $\left(0,11 \mathrm{~m}^{3} / \mathrm{t}\right)$ deve ser encaminhado para a estação de tratamento de efluentes. Como água de lavagem para o estágio $A_{\text {hot }}$, foram utilizados $4 \mathrm{~m}^{3} / \mathrm{t}$ de condensado da evaporação, os quais, somados aos $5 \mathrm{~m}^{3} / \mathrm{t}$ de filtrado oriundos do estágio (EOP), totalizaram um volume de $9 \mathrm{~m}^{3} / \mathrm{t}$. O restante do efluente alcalino $\left(4 \mathrm{~m}^{3} / \mathrm{t}\right)$ procedente do estágio (EOP) foi enviado para a lavagem na prensa da pré- $\mathrm{O}_{2}$. A lavagem da polpa pré- $\mathrm{O}_{2}$ não foi simulada em laboratório. O condensado da evaporação apresentava as seguintes características: $\mathrm{pH}$ de 9,2, DQO de $1.564 \mathrm{mg} \mathrm{O}_{2} / \mathrm{L}$, cor de $183 \mathrm{mg} \mathrm{Pt} / \mathrm{L}$ e TRS (Total Reduced Sulphur - Compostos de enxofre reduzido) de 19,2 $\mathrm{mg} \mathrm{SO}_{2} / \mathrm{L}$.
Como água de lavagem do estágio (EOP), foram utilizados $4 \mathrm{~m}^{3} / \mathrm{t}$ de condensado da evaporação, que somados aos $5 \mathrm{~m}^{3} / \mathrm{t}$ de filtrados provenientes da máquina formadora de celulose (água branca) totalizaram $9 \mathrm{~m}^{3} /$ t. Com essa estratégia, parte significativa do efluente do branqueamento retorna para o sistema de recuperação. Somente $9 \mathrm{~m}^{3} / \mathrm{t}$ de filtrado do estágio D foram totalmente descartados para a estação de tratamento de efluentes. Na simulação em laboratório, a água branca foi substituída por água destilada.

No estágio (PO) foram utilizados como água de lavagem $4 \mathrm{~m}^{3} / \mathrm{t}$ de água branca, inclusive para diluição da polpa $\left(6,67 \mathrm{~m}^{3} / \mathrm{t}\right)$. O mesmo volume de filtrado originado na lavagem da polpa após o estágio (PO) foi usado como água de lavagem no estágio D.

\subsubsection{Lavagem da polpa}

A simulação da lavagem da polpa, utilizando filtro a vácuo, foi efetuada da seguinte forma: a polpa proveniente do estágio de branqueamento efetuado na consistência de $10 \%$ foi diluída para 1,5\% com filtrado do ciclo anterior, exceto no primeiro ciclo, no qual foi utilizado água destilada. Após a diluição, a polpa foi desaguada em coluna e lavada por deslocamento, com o equivalente a $9 \mathrm{~m}^{3} / \mathrm{t}$ de filtrado do ciclo anterior, exceto no primeiro ciclo, em que foi usado água destilada. A remoção de água por vácuo no final da lavagem foi efetuada de forma a manter uma consistência de $12 \%$ na polpa lavada. Tal procedimento foi realizado após cada estágio, exceto no último. Depois do estágio (PO), foi simulada uma lavagem por prensa, tal como previsto na Figura 1. A simulação da lavagem da polpa por prensa 
foi feita da seguinte forma: a polpa proveniente do estágio de dioxidação efetuado na consistência de $10 \%$ foi diluída para $4 \%$ com filtrado do próprio (PO) do ciclo anterior, exceto no primeiro ciclo, no qual foi utilizado água destilada. Após a diluição, a polpa foi desaguada em coluna, lavada por deslocamento com o equivalente a $4 \mathrm{~m}^{3} / \mathrm{t}$ de água destilada e centrifugada até a consistência de $30 \%$.

A primeira amostra de polpa foi lavada com água destilada, sendo os filtrados de todos os estágios da seqüência armazenados. Esses, por sua vez, foram utilizados no ajuste de consistência e lavagem da próxima amostra de polpa, de acordo com o esquema mostrado na Figura 1. Denominou-se ciclo de branqueamento cada uma dessas etapas, simulando o processo industrial. Os ciclos foram repetidos tantas vezes quanto necessário até que os valores de DQO dos filtrados de cada estágio se estabilizassem. As principais condições das etapas de branqueamento são apresentadas no Quadro 2.

\subsubsection{Procedimentos analíticos}

Exceto quando explicitamente descritos, os métodos de análises utilizados foram padrões TAPPI, DIN, PTS, ISO e STANDARD METHODS FOR THE EXAMINATION OF WATER AND WASTEWATER.

Quadro 2 - Condições gerais de branqueamento Table 2 - General bleaching conditions

\begin{tabular}{lcccc}
\hline Condições & \multicolumn{4}{c}{ Estágios de Branqueamento } \\
\cline { 2 - 5 } & $\mathrm{A}_{\text {hot }}$ & $(\mathrm{EOP})$ & $\mathrm{D}$ & $(\mathrm{PO})$ \\
\hline Consistência, \% & 10 & 10 & 10 & 10 \\
Tempo, min & 140 & $15+70$ & 150 & 60 \\
Temperatura, ${ }^{\circ} \mathrm{C}$ & 95 & 85 & 75 & 102 \\
$\mathrm{pH}_{\text {final, }}( \pm$ 0,2 $)$ & 3,5 & 11,0 & 4,5 & 11,0 \\
$\mathrm{MgSO}_{4} .7 \mathrm{H}_{2} \mathrm{O}, \%$ & - & - & - & 0,167 \\
$\mathrm{Pressão}_{\mathrm{rPa}}$ & - & $200+0$ & - & 400 \\
$\mathrm{H}_{2} \mathrm{SO}_{4}, \%$ & 1,2 & - & - & - \\
$\mathrm{NaOH}_{0} \%$ & - & 0,89 & $0,33-0,5$ & 0,89 \\
$\mathrm{H}_{2} \mathrm{O}_{2}, \%$ & - & 0,33 & - & $1,0-1,33$ \\
$\mathrm{ClO}_{2}, \%$ & - & - & 2,78 & - \\
\hline
\end{tabular}

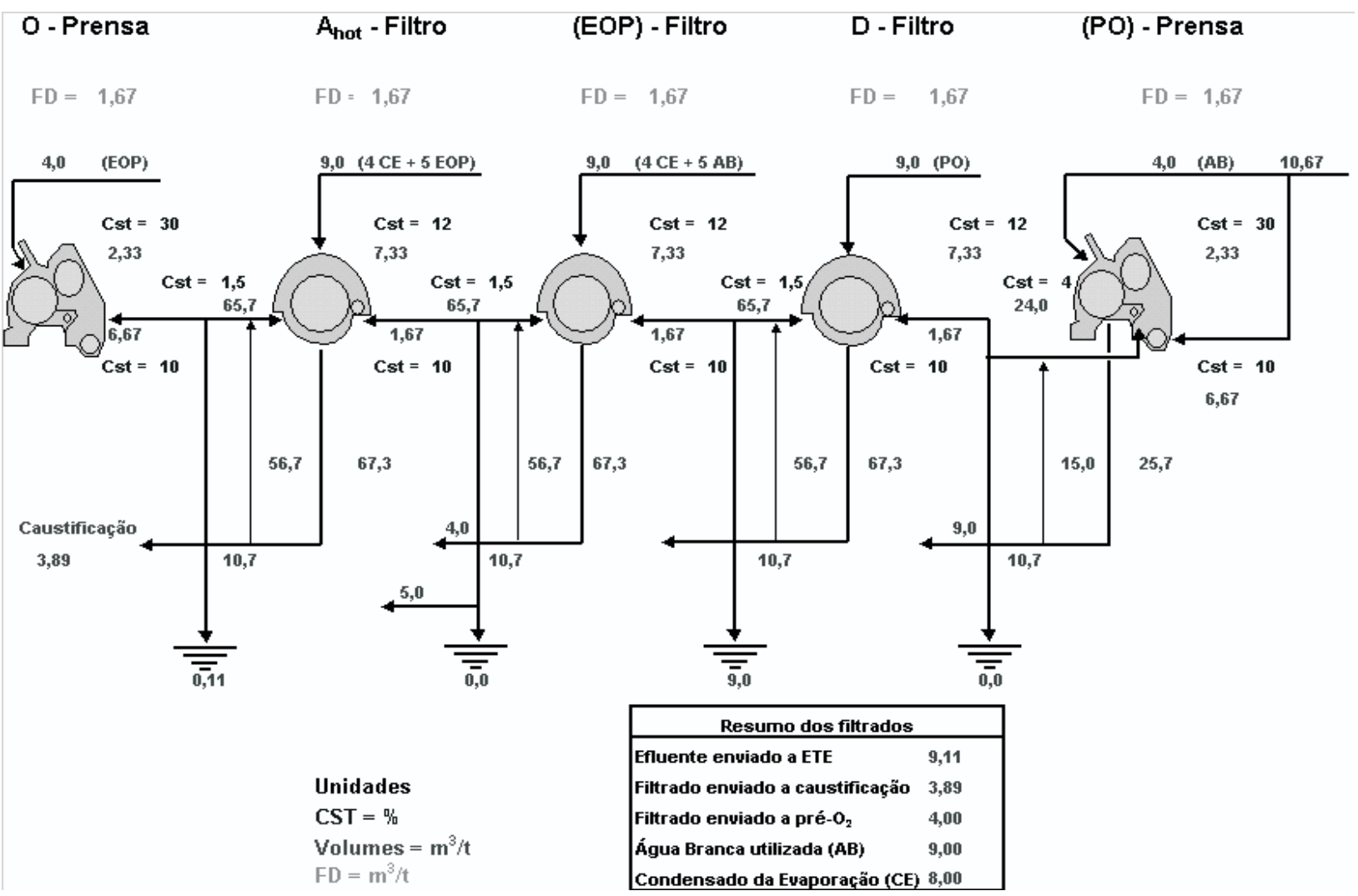

Figura 1 - Esquema simplificado de recirculação de filtrados utilizados na seqüência $A_{\text {hot }}(E O P) D(P O)$. Figure $1-A_{\text {hot }}(E O P) D(P O)$ Bleaching Filtrate Strategy.

R. Árvore, Viçosa-MG, v.30, n.1, p.129-139, 2006 


\section{RESULTADOS E DISCUSSÃO}

\subsection{Estabilidade de DQO dos filtrados}

Como observado na Figura 2, os filtrados dos vários estágios da seqüência $A_{\text {hot }}(\mathrm{EOP}) \mathrm{D}(\mathrm{PO})$ atingiram estabilidade nos valores de DQO somente após o décimo ciclo de branqueamento. Essa estabilidade da concentração de compostos orgânicos mensurada pela DQO significa que quando uma seqüência de branqueamento é repetida, utilizando os filtrados do ciclo anterior, não há variação significativa no valor de DQO dos filtrados. O número de ciclos para atingir valores de DQO constantes, ou em estado estacionário, está estreitamente relacionado com o fator diluição e com o tipo de lavagem empregado. Na lavagem por filtros a vácuo, inicialmente a polpa é diluída para baixa consistência (1,5\%), ocorrendo, portanto, significativa diluição do material orgânico nos filtrados, o que torna o equilíbrio de DQO mais difícil. Neste caso, tornase necessário maior número de ciclos para atingir valores de DQO constantes.

É importante notar que cerca de $8 \mathrm{~m}^{3} / \mathrm{t}$ de condensado da evaporação foram utilizados na lavagem da polpa, sendo distribuídos igualmente na lavagem desta, após os estágios $\mathrm{A}_{\text {hot }}$ e (EOP). A utilização do condensado da evaporação como água de lavagem no branqueamento é necessária em virtude do interesse de se reduzir o volume de efluente a ser tratado além da carga. Geralmente, o condensado pode ser usado na caustificação e no último elemento lavador após pré$\mathrm{O}_{2}$, perfazendo um volume semelhante ao do licor preto fraco enviado à evaporação ( 8 a $\left.9 \mathrm{~m}^{3} / \mathrm{t}\right)$, como mencionado na Figura 1.

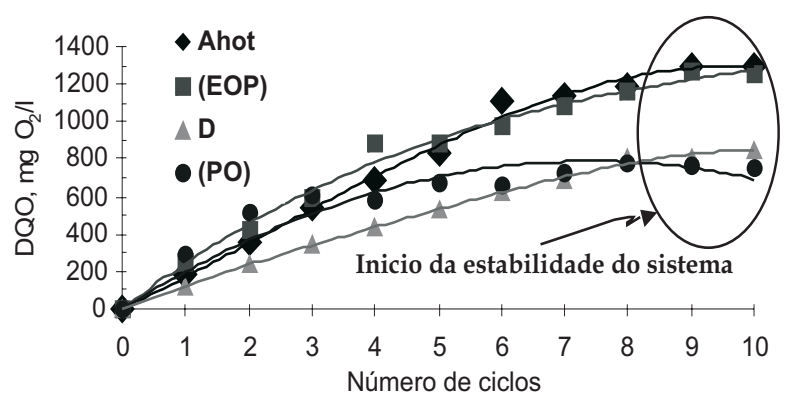

Figura 2 - Comportamento dos valores de DQO em função dos ciclos de branqueamento.

Figure 2 - Behaviour of filtrate COD, stage by stage of the bleaching sequence, as a function of the bleaching cycle.
Os resultados experimentais deste estudo de recirculação dos filtrados de lavagem estão apresentados no Quadro 3 (Anexo). Neste, a alvura final da polpa branqueada estabilizou-se após o segundo ciclo de branqueamento. Tal fato indica que o acúmulo de DQO que ocorreu após o segundo ciclo não teve efeito significativo nessa propriedade da polpa. Como conseqüência da redução da alvura após o primeiro ciclo, houve aumento de $0,33 \%$ na dosagem de peróxido de hidrogênio no estágio (PO). Do segundo ciclo em diante, não houve aumento no requerimento de reagentes químicos. Ao longo dos ciclos, também não houve impacto significativo na viscosidade final da polpa e nem no número kappa após cada estágio, o que resultou numa seletividade semelhante em todos os ciclos.

\subsection{Caracterização dos efluentes da seqüência $\mathrm{A}_{\text {hot }}(\mathrm{EOP}) \mathrm{D}(\mathrm{PO})$}

No Quadro 4, mostram-se as principais características dos efluentes originados da seqüência $\mathrm{A}_{\text {hot }}(\mathrm{EOP}) \mathrm{D}(\mathrm{PO})$, após 10 ciclos de branqueamento. De acordo com o esquema proposto na Figura 1, basicamente se têm dois efluentes da seqüência: os filtrados dos estágios $\mathrm{A}_{\text {hot }}$ e D. A maior fração do efluente enviado à estação de tratamento de efluentes é constituída por filtrado $D$, já que uma fração significativa do filtrado do estágio $\mathrm{A}_{\text {hot }}$ é passível de reutilização.

Observou-se que os pHs dos efluentes dos vários ciclos são semelhantes e próximos da neutralidade. Esse fato se deve à utilização de um filtrado alcalino de um estágio posterior na lavagem da polpa dos estágios $A_{\text {hot }}$ e D, como apresentado na Figura 1. Pode ser observado também, no Quadro 4, que a maior parte da carga orgânica solúvel, medida pelos parâmetros $\mathrm{DQO}, \mathrm{DBO}_{5}$ e cor gerada no branqueamento, é originada dos filtrados do estágio $\mathrm{A}_{\text {hot }}$. Geralmente, maior parte do volume desse filtrado $\left(\sim 6,67 \mathrm{~m}^{3} / \mathrm{t}\right)$ é utilizada no ajuste de consistência de 30 para $10 \%$. O restante ( $\left.4 \mathrm{~m}^{3} / \mathrm{t}\right)$ é passível de recirculação e reaproveitamento, já que apresenta baixos teores de cloretos. Com a reutilização desse filtrado do estágio $A_{\text {hot }}$, parte da carga orgânica deixa de ser enviada para ETE. Portanto, a maior parte do efluente de branqueamento, em volume, a ser enviada à estação de tratamento de efluentes é originada no estágio de dioxidação.

R. Árvore, Viçosa-MG, v.30, n.1, p.129-139, 2006 


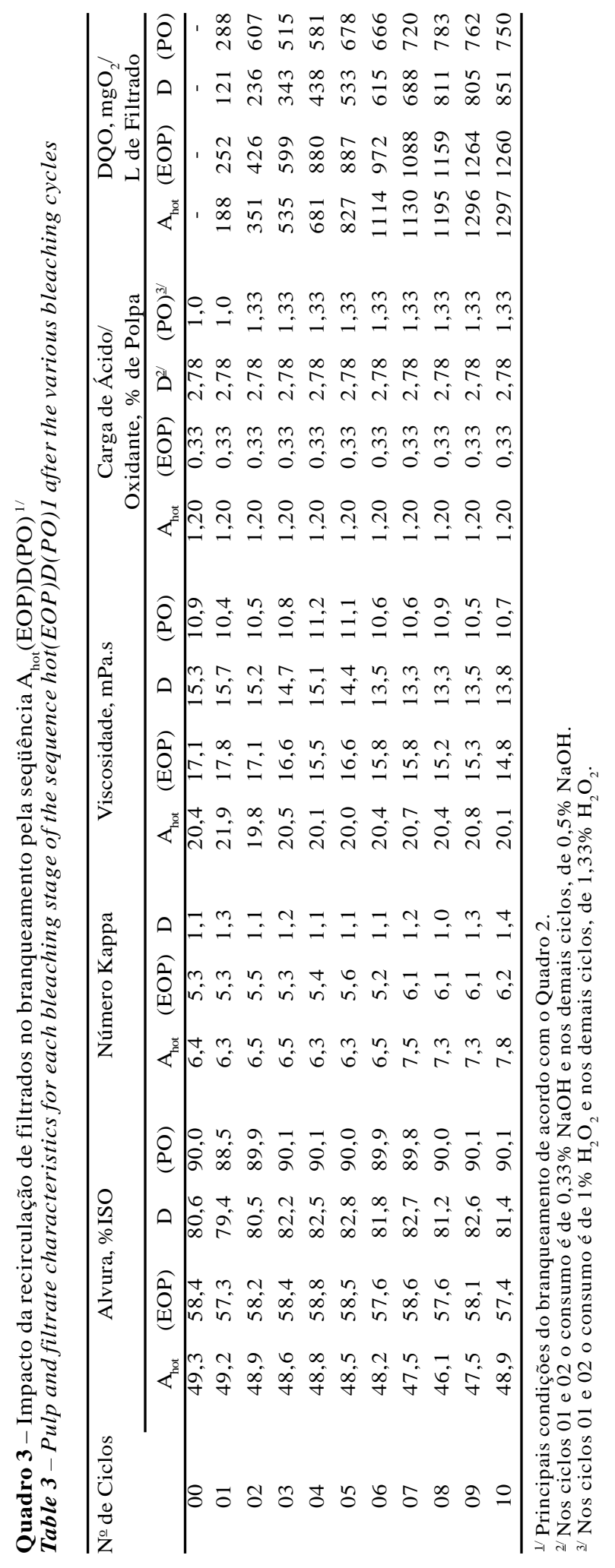

Os níveis de concentração de matéria orgânica indicados pela DQO no filtrado estão numa faixa inferior à dos efluentes atuais da CENIBRA (Quadro 4). É importante observar que o material enviado à ETE, vindo da dioxidação, apresenta excelente biodegradabilidade. Tendo em vista a alta relação $\left(\mathrm{DBO}_{5} / \mathrm{DQO}=0,54\right)$, cerca de 54\% da DQO desse efluente é passível de remoção durante o tratamento biológico, o que é confirmado pela ausência de toxicidade aguda (MICROTOX). A cor do efluente a ser tratado é também muito baixa, o que é vantajoso, já que o tratamento biológico não é eficiente na remoção de cor do efluente industrial. Tal fato indica que esses compostos que promovem a cor do efluente podem ser removidos pelas lavagens dos primeiros estágios de branqueamento. Yousefian e Reeve (2000) mencionaram que esses compostos são de alto peso molecular vindos da lignina residual, os quais apresentam baixa biodegradabilidade diante do tratamento biológico. Esse é o motivo pelo qual a implantação de um estágio de pré- $\mathrm{O}_{2}$ apresenta impacto significativo de redução da cor do efluente industrial. Com essa estratégia de recirculação de filtrados proposta, obtiveram-se resultados de cor abaixo daqueles valores típicos de efluentes tratados biologicamente ou, mesmo, por uma etapa terciária (WÖRSTER et al., 1999). O efluente a ser tratado apresentou também baixo conteúdo de compostos organoclorados (AOX). Esse fato se deve à baixa carga de dióxido de cloro aplicado $(10,56 \mathrm{~kg}$ $\mathrm{ClO}_{2} / \mathrm{t}$ ), aliado ao baixo número kappa da polpa que entra na dioxidação.

O valor de sólidos solúveis voláteis (SSV) listado no Quadro 4 indica o impacto global dos inertes orgânicos do filtrado do estágio $\mathrm{A}_{\text {hot }}$. Levando em consideração o volume que pode ser usado na caustificação (3,89 $\mathrm{m}^{3} / \mathrm{t}$ ) e o valor desses orgânicos solúveis mensurado no filtrado do estágio $A_{\text {hot }}$, tem-se um valor em torno de 3,77 kg SSV/t, os quais, por sua vez, podem permanecer na lama de cal ou no licor branco fraco, que dissolve o smelt no tanque de dissolução. Neste último caso, o impacto dos NPEs (orgânicos) no sistema se dá no sistema de remoção de dregs, bem como no licor branco.

Além da carga orgânica dos filtrados a serem recirculados, devem ser levados em consideração alguns inorgânicos solúveis em meio alcalino. Dentre os principais, os íons de cloreto e de potássio se acumulam continuamente no ciclo do elemento sódio, que se concentram em maior intensidade na cinza do precipitador eletrostático, sendo este um ponto estratégico para 
a sua remoção (TRAN et al., 1990). Existe uma série de processos disponíveis no mercado que apresenta eficiência aceitável na remoção de cloreto e potássio da cinza do precipitador eletrostático (MAPLES et al., 1994; SHENASSA et al., 1996; PFROMM e RAPP, 1997; JEMAA et al., 1998; MINDAY et al., 1998). De modo geral, os principais problemas causados pela alta concentração de cloretos e potássio no ciclo de recuperação se resumem em entupimento e corrosão nas tubulações da caldeira, bem como na contínua recirculação desses inertes no processo (HUPA et al., 1997). Portanto, a partir de certos níveis, a reutilização de filtrados que contêm íons geralmente requer a implantação de processos específicos para removêlos ou, mesmo, de purgas da cinza do precipitador eletrostático. Tran et al. (1990) relataram que níveis normais dos íons Cl e K são, respectivamente, valores

Quadro 4- Principais características dos efluentes originados da seqüência $\mathrm{A}_{\text {hot }}(\mathrm{EOP}) \mathrm{D}(\mathrm{PO})$

Table 4-Overall characteristics of Ahot $(E O P) D(P O)$ bleaching effluent, extracted after the tenth bleaching cycle

\begin{tabular}{|c|c|c|}
\hline \multirow[t]{2}{*}{ Parâmetros Analisados } & \multicolumn{2}{|c|}{$\begin{array}{l}\text { Efluentes do } 10^{\circ} \text { Ciclo da } \\
\text { Sequência } A_{\text {hot }}(\text { EOP }) D(P O)^{*}\end{array}$} \\
\hline & $A_{\text {hot }}$ & $\bar{D}$ \\
\hline$\overline{\mathrm{pH}}$ & 6,7 & 6,7 \\
\hline $\begin{array}{l}\text { Toxicidade aguda } \\
\text { (MICROTOX) EC }\end{array}$ & $>100$ & $>100$ \\
\hline $\begin{array}{l}\mathrm{DQO}, \mathrm{mg} \\
\mathrm{O}_{\Omega} / \mathrm{l}\left(\mathrm{kg} \mathrm{O}_{/} / \mathrm{t}\right)\end{array}$ & $1.297(11,67)$ & $851(7,66)$ \\
\hline $\mathrm{DBO}_{5}, \mathrm{mg} \mathrm{O}_{2} / 1$ & 670 & 456 \\
\hline Biodegradabilidade, $\mathrm{DBO}_{5} / \mathrm{DQO}$ & 0,52 & 0,54 \\
\hline Cor, mg Pt/l & 1.356 & 94,0 \\
\hline $\mathrm{AOX}, \mathrm{mg} \mathrm{Cl}^{-} / \mathrm{l}$ & 0,6 & 5,8 \\
\hline $\begin{array}{l}\text { Sólidos Solúveis Voláteis } \\
\text { (SSV), mg/l }\end{array}$ & 968 & 635 \\
\hline$\overline{\text { Inorgânicos, mg/l }}$ & & \\
\hline $\mathrm{Na}$ & 594 & 402 \\
\hline $\mathrm{SO}_{4}=$ & 1.172 & 51,4 \\
\hline $\mathrm{Cl}$ & 66,5 & 338 \\
\hline $\mathrm{K}$ & 54,7 & 7,0 \\
\hline $\mathrm{Ca}$ & 31,3 & 3,87 \\
\hline Mg & 2,42 & 1,33 \\
\hline $\mathrm{Fe}$ & 0,04 & 0,04 \\
\hline $\mathrm{Cu}$ & 0,04 & 0,02 \\
\hline Mn & 0,86 & 0,30 \\
\hline $\mathrm{P}$ & 4,66 & 3,78 \\
\hline $\mathrm{Al}$ & 1,02 & 0,82 \\
\hline Insolúveis em $\mathrm{HCl}$ & 9,33 & 7,33 \\
\hline
\end{tabular}

* Características médias do efluente industrial da CENIBRA vindo da seqüência D(EOP)DD: DQO de $1.074 \mathrm{mg} \mathrm{O} / \mathrm{l}$, AOX de 0,49 mg $\mathrm{Cl}^{-} / \mathrm{t}$ e $\mathrm{EC}_{50 \%}$ de 20,3 . inferiores a $7 \%$ e entre 1 e $5 \%$, base sólidos secos do licor preto concentrado. Como mostrado no Quadro 4, o alto conteúdo de íons $\mathrm{Cl}$ no filtrado do estágio (D) é o principal constituinte químico que dificulta a reutilização desse filtrado, em circuito fechado.

Além dos íons cloreto e potássio, outros NPEs, também solúveis em meio alcalino, são os íons de $\mathrm{P}$ e Si. Esses, por sua vez, tendem a se acumular no sistema de recuperação na forma de compostos inorgânicos, principalmente no ciclo do cálcio, aumentando a carga morta na circulação da lama de cal (ULMGREN, 1997). É bem conhecido o efeito que os íons de P e Si têm na perda de eficiência da reação de calcinação do forno. Em torno de 1\% (base peso) de Si e de P na lama decresce a CaO útil, respectivamente, em torno de 6 e $5 \%$ (base peso). A sílica também tem alta solubilidade no licor branco, em torno de $15 \mathrm{mmol} / \mathrm{L}$, como íons silicato $\mathrm{SiO}_{2}(\mathrm{OH})_{2}{ }^{2}$, sendo uma solubilidade alta devido ao fato de o meio estar básico, podendo acumular-se no circuito. $\mathrm{Na}$ presença de concentrações superiores à da solubilidade dos íons silicato é formado o silicato de cálcio $\left(\mathrm{CaSiO}_{3}\right)$ na caustificação, que sai na forma de grits na etapa de extinção da cal (slaking) (ULMGREN, 1996). Segundo Vältilä et al. (1996), a sílica pode ser removida com eficiência pela adição de magnésio se a relação molar $\mathrm{Mg} / \mathrm{Si}$ estiver em torno de 5 a 6 e o pH for maior que 10,5. Já o fosfato de cálcio é acumulado no ciclo da lama, pois este é muito mais solúvel no licor verde do que no licor branco (ULMGREN, 1996, 1997). Segundo Gleadow et al. (1997), uma das formas de controle desse ENP se dá pela purga da lama de cal, com posterior reposição de calcário ou cal.

Existem, no entanto, outros NPEs insolúveis em meio alcalino. O Al e o Mg, salvo algum distúrbio significativo nas suas proporções, são removidos simultaneamente do sistema. Esses elementos, mais o carbonato, formam um composto insolúvel em meio alcalino denominado hydrotalcite $-\mathrm{Mg}_{1-\mathrm{x}} \mathrm{Al}(\mathrm{OH})_{2}\left(\mathrm{CO}_{3}\right)_{\mathrm{x} /}$ ${ }_{2}{ }^{*} \mathrm{nH}_{2} \mathrm{O}_{(\mathrm{s})}$, em que $x$ varia de 0,1 a 0,34 (ULMGREN, 1997). Esse composto pode sair através da decantação ou filtração dos dregs, juntamente com outros NPEs que formam sais pouco solúveis em meio alcalino (Fe, $\mathrm{Mn}, \mathrm{Cu}$ e etc.). Portanto, um dos principais pontos de remoção dos NPEs do processo se dá pela eficiente purga de dregs (ULMGREN, 1996; 1997). Visando aumentar a eficiência de remoção de dregs do sistema, têm-se, basicamente, três opções: (1) adição de floculantes apropriados, (2) adição de cal e (3) filtração (GLEADOW et al., 1997).

R. Árvore, Viçosa-MG, v.30, n.1, p.129-139, 2006 
Entre os inorgânicos solúveis no filtrado do estágio $\mathrm{A}_{\text {hot }}$ existem elementos de valor econômico. Os íons $\mathrm{Na} \mathrm{e} \mathrm{SO}_{4}{ }^{=}$presentes no filtrado devem ser considerados como elementos de reposição, já que fazem parte do “make up" do sistema de recuperação dos produtos químicos do processo kraft.

\subsection{Reutilização do filtrado do estágio $A_{h o t}$}

Com o crescente aumento do ritmo de produção, em praticamente todas as fábricas, para uma mesma capacidade instalada dos equipamentos e, principalmente, dos elementos lavadores da linha de fibra, tem-se como fato o incremento da perda alcalina. Outro fato importante a ser mencionado, e de difícil gerenciamento, está associado às tendências mercadológicas. No último ano, ocorreu uma crescente elevação no preço do hidróxido de sódio. A Figura 3 ilustra o aumento do custo operacional por tonelada de celulose, em função do incremento da perda alcalina e do custo do hidróxido de sódio, nesse período. Observase, nessa figura, que existe um incremento significativo de 1999 para 2001. Assim, o custo de produção das linhas 1 e 2 é de 5,47 e 3,40 US\$/t, respectivamente, devido à reposição do hidróxido de sódio, tendo como base, exclusivamente, a perda alcalina estimada após a prensa da pré- $\mathrm{O}_{2}$ do período do ano de 2001 e o custo do hidróxido de sódio no ano de 2001.

Qualquer alternativa, portanto, que resulte em recuperação do elemento sódio no processo tem impacto significativo no custo de produção. Dessa forma, como previsto na Figura 1, a recirculação e o reaproveitamento do filtrado do estágio $A_{\text {hot }}$, estágio esse posicionado após o estágio da pré- $\mathrm{O}_{2}$, são alternativas eficientes de recuperação do elemento sódio no processo. Esse fato é sem dúvida a principal justificativa econômica de se recircular o efluente oriundo do $A_{\text {hot }}$ com possibilidade de recuperar cerca de $67 \%$ do hidróxido de sódio do “make up” (Figura 4). É importante observar também que, somado à recuperação de parte do elemento sódio, tem-se um decréscimo significativo do consumo de água industrial e de geração de efluentes. Paralelamente à redução do volume de efluente, tem-se a redução da carga de DQO, DBO, cor e AOX, gerados na planta de branqueamento. Esses fatores deverão ter impacto significativo num futuro próximo, com a cobrança pelo uso da água e pelo lançamento de efluentes com a nova legislação em vigor no Brasil.

\section{4. $A_{\text {hot }}$ versus $D_{\text {hot }}$ no primeiro estágio}

Se no primeiro estágio de branqueamento for adicionado dióxido de cloro, tal como previsto na tecnologia $\mathrm{D}_{\text {hot }}$, os benefícios citados no item anterior não serão alcançados, além do evidente incremento de temperatura do efluente a ser tratado. A favor da utilização do $\mathrm{D}_{\text {hot }}$ citam-se a redução da carga de ácido sulfúrico para ajustar o $\mathrm{pH}$ dessa etapa e a redução no consumo de peróxido de hidrogênio. Na Figura 5, mostra-se um comparativo dos custos estimados dos reagentes químicos de branqueamento para ambas as tecnologias, comparados com os da seqüência D(EOP)DD (COSTA et al., 2001). Os principais resultados, estágio por estágio, das seqüências referência, $\mathrm{D}_{\text {hot }}(\mathrm{EOP}) \mathrm{D}(\mathrm{PO})$

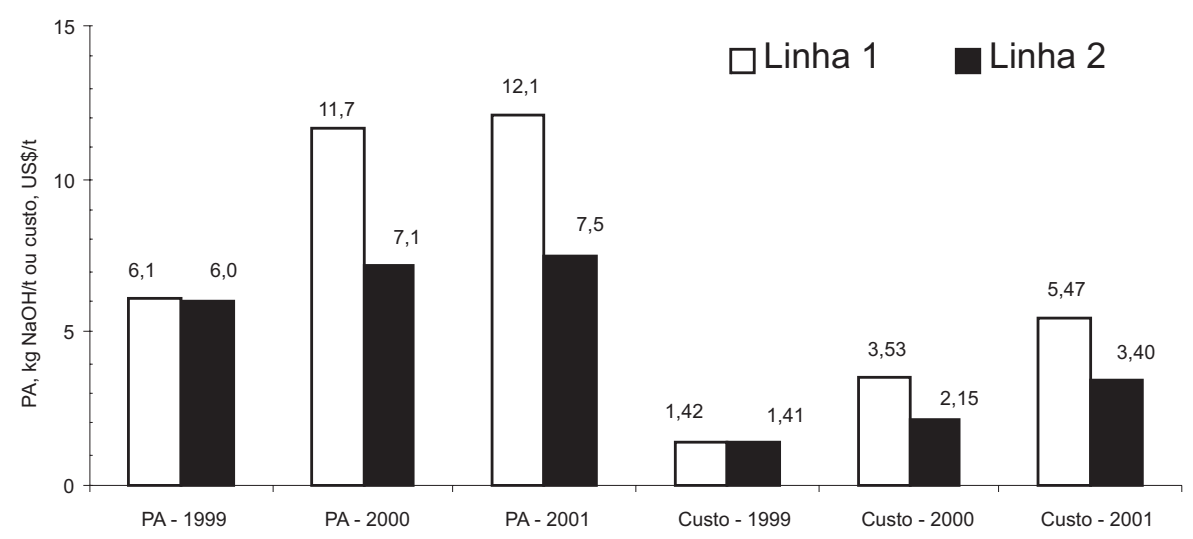

Figura 3 - Valores médios estimados de perda alcalina e do custo de produção correspondente ao período de 1999 a 2001. Figure 3 - Average values of sodium loss (SL) and production costs (US\$) for CENIBRA's Line 1 and 2 in the period of 1999-2001.

R. Árvore, Viçosa-MG, v.30, n.1, p.129-139, 2006

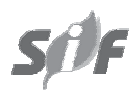


e $A_{\text {hot }}(E O P) D(P O)$, para a polpa F (Quadro 1), estão listados no Quadro 5. Para reposição de hidróxido de sódio, levou-se em consideração a realidade da CENIBRA da linha 1 no ano de 2000, cuja perda alcalina média foi de $11,7 \mathrm{~kg} \mathrm{NaOH} / \mathrm{t}$ e o custo do hidróxido de sódio, de aproximadamente US\$ 453/t. Considerou-se também a redução de metade do consumo de água $\left(9 \mathrm{~m}^{3} / \mathrm{t}\right)$, tendo como conseqüência um mesmo decréscimo na geração de efluentes.

Em decorrência do incremento do preço do hidróxido de sódio no último ano, pode ser observado, na Figura 5 , que a recuperação do elemento sódio intensifica as diferenças de custo operacional dos produtos

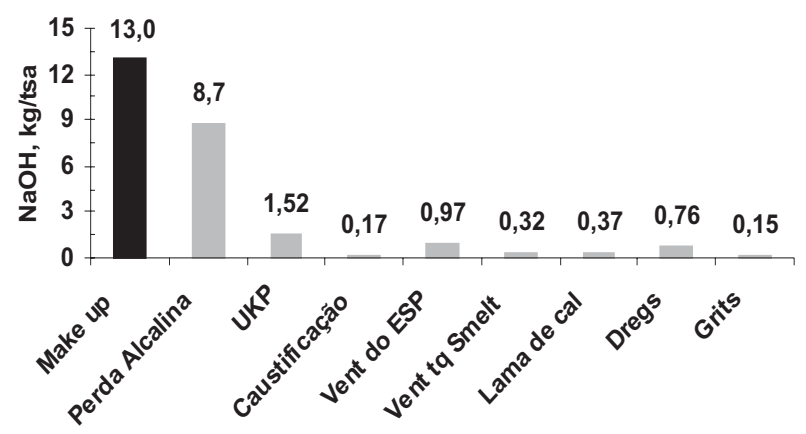

Figura 4 - Balanço do elemento sódio no processo durante o ano de 2001.

Figure 4 -Mill sodium make up balance during the year of 2001.

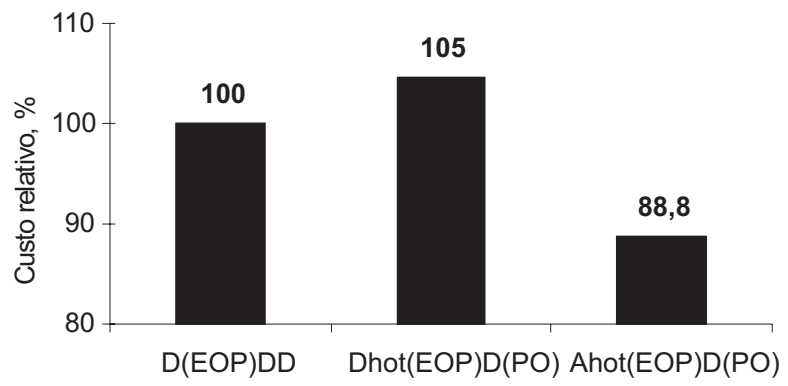

Figura 5 - Comparação dos custos dos produtos químicos de branqueamento entre as tecnologias $\mathrm{A}_{\text {hot }}$ e $\mathrm{D}_{\text {hot }}$, de acordo com o consumo dos produtos químicos.

Figure 5 - Comparative analysis for total bleaching costs (reagents, sodium make up and effluent treatment) between the Ahot and Dhot technologies having as reference the sequences $D(E O P) D D$. químicos. Conclui-se, por essa figura, que existe uma diferença de $16,2 \%$ a favor da tecnologia $\mathrm{A}_{\text {hot }}$, em relação à $\mathrm{D}_{\text {hot }}$. Nesse contexto, do ponto de vista de custo operacional dos produtos químicos de branqueamento, não resta dúvida de que a tecnologia do $\mathrm{A}_{\text {hot }}$ é superior à tecnologia do $\mathrm{D}_{\text {hot }}$. Portanto, os principais diferenciais entre elas são a possibilidade de fechamento de circuito com o estágio $\mathrm{A}_{\text {hot }}$ e a recuperação do hidróxido de sódio contido no efluente do estágio $\mathrm{A}_{\text {hot }}$. Basicamente, existem duas formas de retornar esse filtrado ao sistema de recuperação, que não exigem ampliação da capacidade de evaporação: (1) lavando em contracorrente a polpa pré- $\mathrm{O}_{2}$ e (2) enviando o filtrado do estágio $\mathrm{A}_{\text {hot }}$ para a caustificação.

A opção de enviar o filtrado ácido em contracorrente para lavar a polpa pré- $\mathrm{O}_{2}$ tem como principais desvantagens os seguintes pontos: (a) acréscimo do consumo de hidróxido de sódio no estágio da pré- $\mathrm{O}_{2}$; (b) alto risco de formação de oxalato de cálcio na interface do estágio $\mathrm{A}_{\text {hot }}$ com o estágio anterior da pré- $\mathrm{O}_{2}$, em função da presença de oxalato e de cálcio vindo, respectivamente, da pré- $\mathrm{O}_{2}$ e do $\mathrm{A}_{\text {hot }}$, aliados ao decréscimo de $\mathrm{pH}$ para a faixa de 2 a 8, propiciando maior formação de oxalato de cálcio (ULMGREN, 1997; COSTA et al., 1999); (c) provável recirculação de metais pesados solubilizados durante $A_{\text {hot }}$ sob condições ácidas a quente; (d) recirculação com possibilidade de reprecipitação de lignina residual, proveniente do carryover da pré- $\mathrm{O}_{2}$ e, ou, de estruturas hidrolisáveis da lignina residual em meio ácido a quente; e (e) recirculação dos derivados furanosídicos (2-furóico e 5-formil-2furóico), originados da hidrólise ácida dos HexAs.

A alternativa de reutilização do filtrado ácido como água industrial na caustificação tem como desvantagens: (a) necessita de previa filtração do efluente, com a finalidade de remoção das fibras; (b) incremento do conteúdo de NEPs, tanto na lama de cal a ser calcinada quanto no licor verde a ser clarificado. No entanto, é desnecessária a utilização de hidróxido de sódio para correção de $\mathrm{pH}$. O volume de efluente a ser usado na área da caustificação é igual ao volume do licor branco de polpação, que é cerca de 3,89 m³/t. Desses volumes, 2,78 m³/t são geralmente utilizados na lavagem da lama de cal, enquanto o restante é usado para lavagem de dregs e outras aplicações na caustificação Figura 1.

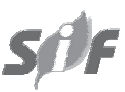

R. Árvore, Viçosa-MG, v.30, n.1, p.129-139, 2006 
Quadro 5 - Principais resultados do branqueamento das seqüências D(EOP)DD, $\mathrm{A}_{\text {tot }}(\mathrm{EOP}) \mathrm{D}(\mathrm{PO})$ e $\mathrm{D}_{\text {hot }}(\mathrm{EOP}) \mathrm{D}(\mathrm{PO})$ Table 5 - Stage by stage bleaching results with the sequences $D(E O P) D D$, Ahot $(E O P) D(P O)$ and $\operatorname{Dhot}(E O P) D(P O)$

\begin{tabular}{|c|c|c|c|c|c|c|c|c|c|c|c|c|}
\hline \multirow{2}{*}{$\begin{array}{l}\text { Condições e } \\
\text { Resultados }\end{array}$} & \multicolumn{12}{|c|}{ Estágio de Branqueamento } \\
\hline & $\mathrm{D}$ & (EOP) & $\mathrm{D}$ & $\mathrm{D}$ & $A_{\text {hot }}$ & $(\mathrm{EOP})$ & $\mathrm{D}$ & $(\mathrm{PO})$ & $\mathrm{D}_{\text {hot }}$ & (EOP) & $\mathrm{D}$ & $(\mathrm{PO})$ \\
\hline Consistência, \% & 10 & 10 & 10 & 10 & 10 & 10 & 10 & 10 & 10 & 10 & 10 & 10 \\
\hline Temperatura, ${ }^{\circ} \mathrm{C}$ & 50 & 85 & 75 & 75 & 95 & 85 & 75 & 102 & 95 & 85 & 75 & 102 \\
\hline Tempo, min & 40 & $15+70$ & 150 & 150 & 140 & $15+70$ & 150 & 60 & 140 & $15+70$ & 150 & 60 \\
\hline Pressão inicial, kPa & - & $200+0$ & - & - & - & $200+0$ & - & 400 & - & $200+0$ & - & 400 \\
\hline $\mathrm{H}_{2} \mathrm{O}_{2}, \%$ & - & 0,333 & - & - & - & 0,333 & - & 1,0 & - & 0,333 & - & 0,57 \\
\hline $\mathrm{O}_{2}, \%$ & - & 0,333 & - & - & - & 0,333 & - & - & - & 0,333 & - & - \\
\hline $\mathrm{NaOH}, \%$ & - & 1,0 & - & 0,166 & - & 0,888 & 0,311 & 0,888 & - & 1,0 & - & 0,888 \\
\hline $\mathrm{MgSO}_{4} \cdot 7 \mathrm{H}_{2} \mathrm{O}, \%$ & - & - & - & - & - & - & - & 0,166 & - & - & - & 0,166 \\
\hline $\mathrm{H}_{2} \mathrm{SO}_{4}, \%$ & 1,4 & - & 0,1 & - & 1,4 & - & - & - & 0,9 & - & - & - \\
\hline $\mathrm{ClO}_{2}, \%$ & 0,570 & - & 0,845 & 0,461 & - & - & 0,85 & - & 0,36 & - & 0,49 & - \\
\hline $\mathrm{pH}_{\text {inicial }}$ & - & 11,7 & - & - & 3,5 & 11,7 & - & - & - & - & & - \\
\hline$\overline{\mathrm{pH}}$ final & 3,0 & 11,1 & 3,4 & 4,3 & 3,4 & 11,5 & 4,3 & 10,4 & 3,2 & 11,5 & 3,9 & 10,7 \\
\hline Rendimento, \% & 99,1 & 98,0 & 99,1 & 99,5 & 98,8 & 99,1 & 98,5 & 98,3 & 98,6 & 97,9 & 99,7 & 99 \\
\hline Consumo de oxidante, \% & 100 & 100 & 100 & 100 & - & 100 & 100 & 80 & 100 & 100 & 100 & 79,1 \\
\hline Alvura, A.D. \% ISO & 64,6 & 77,6 & 87,2 & 90 & 50,8 & 60,3 & 81,1 & 89,9 & 57,6 & 74,7 & 85,1 & 90,0 \\
\hline Alvura, O.D. \% ISO & - & - & - & 87,3 & - & - & - & 88,3 & - & - & - & 88,4 \\
\hline Número kappa & - & 4,2 & - & - & 5,3 & 5,1 & 1,5 & - & - & 3,2 & - & - \\
\hline Viscosidade, mPa.s & - & 14,9 & 14,0 & 13,4 & 18,5 & 15 & - & 11,5 & - & 13,6 & 13,1 & 11,4 \\
\hline \multicolumn{6}{|c|}{$\begin{array}{ll}\mathrm{ClO}_{2} \text { total, } \mathrm{kg} / \mathrm{t} & 1,876\end{array}$} & \multicolumn{2}{|c|}{0,845} & & \multicolumn{2}{|c|}{0,850} & & \\
\hline \multicolumn{3}{|l|}{$\mathrm{H}_{2} \mathrm{O}_{2}^{2}$ total, kg/t } & \multicolumn{3}{|c|}{0,333} & \multicolumn{2}{|c|}{1,333} & & \multicolumn{2}{|c|}{0,900} & & \\
\hline \multicolumn{3}{|c|}{ OX total da polpa, $\mathrm{g} \mathrm{Cl}^{-} / \mathrm{t}$} & \multicolumn{2}{|c|}{133} & & \multicolumn{2}{|c|}{24,1} & & \multicolumn{2}{|c|}{53,0} & & \\
\hline \multicolumn{3}{|c|}{ AOX total do efluente, $\mathrm{kg} \mathrm{Cl}^{-} / \mathrm{t}$} & \multicolumn{2}{|c|}{1112} & & \multicolumn{2}{|c|}{277} & & \multicolumn{2}{|c|}{554} & & \\
\hline DQO total, $\mathrm{kg} \mathrm{O}_{2} / \mathrm{t}$ & & & \multicolumn{2}{|c|}{35,2} & & \multicolumn{2}{|c|}{39,6} & & \multicolumn{2}{|c|}{38,1} & & \\
\hline $\mathrm{DBO}_{5}$ total, $\mathrm{kg} \mathrm{O}_{2} / \mathrm{t}$ & & & \multicolumn{2}{|c|}{10,8} & & \multicolumn{2}{|c|}{18,2} & & \multicolumn{2}{|c|}{16,8} & & \\
\hline Cor total, $\mathrm{kg} \mathrm{Pt/t}$ & & & \multicolumn{2}{|c|}{34,1} & & \multicolumn{2}{|c|}{44,4} & & \multicolumn{2}{|c|}{33,8} & & \\
\hline
\end{tabular}

\section{CONCLUSÕES}

Neste trabalho, as principais conclusões foram as seguintes:

1. A DQO nos filtrados da seqüência de branqueamento $\mathrm{A}_{\text {hot }}(\mathrm{EOP}) \mathrm{D}(\mathrm{PO})$ estabilizou-se no décimo ciclo de reutilização dos filtrados.

2. O incremento de DQO, devido à recirculação dos filtrados, resultou em aumento do consumo de $3,33 \mathrm{~kg} \mathrm{H}_{2} \mathrm{O}_{2} / \mathrm{t}$ no estágio (PO), após o $2^{\circ}$ ciclo, tendo o consumo permanecido estável após o $3^{\circ}$ ciclo.

3. A utilização do estágio $A_{\text {hot }}$ como primeira etapa de branqueamento mostrou-se mais viável que $\mathrm{D}_{\text {hot }}$ devido ao parcial fechamento de circuito dos filtrados de branqueamento.

4. A estratégia de recirculação de filtrados utilizada reduziu o volume de filtrado de branqueamento em cerca de $9 \mathrm{~m}^{3} / \mathrm{t}$.

5. A reutilização do filtrado do estágio $A_{\text {hot }}$ na caustificação, em substituição aos filtrados usados na lavagem da lama de cal e do dregs, é mais indicada do que reutilizá-lo na lavagem da polpa pré- $\mathrm{O}_{2}$. O impacto dos NEPs (quer seja orgânicos ou inorgânicos) no ciclo do cálcio é pequeno.

6. A reutilização do filtrado $A_{\text {hot }}$ é uma medida economicamente justificável, além de ambientalmente mais correta.

7. O efluente resultante, ou seja, dos estágios D(PO), apresenta baixa carga de DQO, AOX, cor e boa biodegradabilidade.

\section{REFERÊNCIAS BIBLIOGRÁFICAS}

COSTA, M.M. et al. Branqueamento ECF de baixo investimento para a produção de 1 milhão adt/ano na CENIBRA. In: CONGRESSO IBEROAMERICANO DE INVESTIGACION EN CELULOSA Y PAPEL, 2000, Iguazu, Argentina. Proceedings... Iguazu: CIADICYP, 2000. Não paginado. 
COSTA, M.M. et al. Processo de branqueamento com estágio de hidrólise ácida a quente $\left(\mathrm{A}_{\mathrm{hot}}\right)$ para polpa Kraft- $\mathrm{O}_{2}$ de Eucalyptus spp. In: CONGRESSO ANUAL DE CELULOSE E PAPEL DA ABTCP, 34., 2001, São Paulo. Anais... São Paulo: ABTCP: São Paulo, 2001. Não paginado.

GLEADOW, P. et al. Towards closed-cycle kraft: ECF versus TCF case studies. Pulp Paper Canadian, v. 98, n. 4, p. T100-179, 1997.

HUPA, M.; FREDERICK, J. Recovery boiler chemistry. In: TERRY, N.A. (Ed.) Kraft recovery boilers. Atlanta: TAPPI, 1997. p. 41-57.

JEMAA, N. et al. Chloride removal from the kraft recovery boiler ESP dust using the precipitador dust purification (PDP) system. In: INTERNATIONAL CHEMICAL RECOVERY CONFERENCE, 1998. Proceedings... Atlanta: TAPPI, 1998. p. 141-156.

LINDBERG, H.; ENGDAHL, H.; PUUMALINEN, R. Strategies for metal removal control in closed cycle mills. In: INTERNATIONAL PULP BLEACHING CONFERENCE, 1994.

Proceedings... Vancouver: 1994. Não paginado.

MAPLES, G.E. et al. A new process toward bleach plant closure. Tappi Journal, v. 77, n. 11, p.71, 1994.

MINDAY, A.M. et al. An overview of various strategies for balancing salt cake, chloride and potassium levels in an ECF kraft mill. In: INTERNATIONAL CHEMICAL RECOVERY CONFERENCE, 1998. Proceedings... Atlanta: TAPPI, 1998. CD-ROM.

PFROMM, P.H.; RAPP, H. Selective chloride removal from the kraft process: field test. Atlanta: Institute of Paper Science and Technology, 1997. CD-ROM.

SHENASSA, R. et al. Chloride and potassium control in the closed kraft mill liquor cycles. Pulp Paper Canadian, v. 97, n. 5, p.T173-179, 1996.
STANDARD Methods, Examination of Water and Wastewater. 18. ed. Washington, 1998 APHA/ AWWA/ WEF, 1998. 1193p.

TECHNICAL ASSOCIATION OF THE PULP AND PAPER INDUSTRY, TAPPI Standard Methods. Atlanta: TAPPI, and TECHNICAL ASSOCIATION OF POLPING AND PAPER.,[s.d.]. 1145p.

TRAN, H.N.; BARHAM, D.; REEVE, D.W. Chloride and potassium in the kraft chemical recovery cycle. Pulp Paper Canadian, v.91, n. 5, p. T185-190, 1990.

ULGREN, P. Non-process elements in a bleached kraft pulp mill with a high degree of system closure-state of the art. Nord Pulp Paper Research Journal, v. 12, n. 1, p.32-41, 1997.

ULGREN, P. Non-process elements in a bleached kraft pulp mill with increased system closure. In: TAPPI MINIMUM EFFLUENT MILLS SYMPOSIUM, 1996, Atlanta. Proceedings... Atlanta: TAPPI, 1996. p.17-26.

VÄLTTILÄ, O. et al. Removal of silica from bleach plant filtrates. In: TAPPI MINIMUM EFFLUENT MILLS SYMPOSIUM, Atlanta, 1996. Proceedings... Atlanta: TAPPI, 1996. p.309-315.

WÖRSTER, H.E.; COSTA, M.M.; COLODETTE, J.L. Tratamento terciário de efluentes de fábricas de polpa kraft branqueada de eucalipto com ozônio - um estudo de laboratório. Revista Árvore, v.23, n.2, p.233-240, 1999.

YOUSEFIAN, S.; REEVE, D.W. Classes of compounds responsible for COD and colour in bleached kraft mill effluents. In: INTERNATIONAL ENVIRONMENTAL CONFERENCE, 2000, Denver. Proceedings... Denver: TAPPI-PAPTAC, 2000. Não paginado. 